\title{
SUBCONJUNCTIVAL ADMINISTRATION OF SOFRAMYCIN IN THE TREATMENT OF CORNEAL INFECTIONS*
}

\author{
BY \\ D. AINSLIE AND J. E. CAIRNS \\ London
}

THE properties of Soframycin, an antibiotic extracted from the mould Streptomyces decaris, were first evaluated by Massenat-Derouche (1954). Its properties in relation to the eye and its effect upon experimentally produced corneal infections were described by Ainslie and Henderson (1958). It was found to be especially effective against staphylococcal infections but was active too against many Gram-negative bacilli.

While Soframycin may be used topically as an ointment or drops in the treatment of superficial ocular infections, it has no particular advantage in this respect over other broad-spectrum antibiotics. It appeared to be almost completely non-irritant when injected subconjunctivally in the rabbit in doses of up to $500 \mathrm{mg}$., and it was this lack of irritation following experimental subconjunctival injection that made it seem ideal for administration by this route in clinical practice.

In the following series, thirty cases of corneal infection treated by subconjunctival injection of Soframycin are described. The Soframycin was dissolved in either distilled water or $1 / 5000$ adrenaline $(400 \mathrm{mg} . / \mathrm{ml}$.). 100 to $500 \mathrm{mg}$. were given in a single dose and in some cases this was repeated daily for 3 days. Befinite clinical evidence of corneal infection was present in every case, though bacteriological culture was positive in only the minority. Bacteriological cultures gave the following results:
Staph. aureus, 3
Staph. albus, 1
B. coli, 1
Ps. pyocyanea, 1
Pneumococcus, 2
B. xerosis, 3

No growth, 19.

The high proportion of cases in which no growth was obtained is probably explained by the fact that topical antibiotics or chemotherapeutic agents had previously been applied in almost every case. It seems likely that the surface organisms had been destroyed although the deeper infection remained unaffected.

In sixteen of the cases in this series the infection followed injury to the cornea by a foreign body; in twelve there was no history of trauma; in one infection followed a simple corneal abrasion; in the last case infection followed a penetrating corneal wound (Table, overleaf). 
TABLE

\section{RESULTS IN THIRTY CASES OF CORNEAL INFECTION} TREATED WITH SOFRAMYCIN

\begin{tabular}{|c|c|c|c|c|}
\hline $\begin{array}{l}\text { Case } \\
\text { No. }\end{array}$ & Nature of Case & Bacteriology & $\begin{array}{c}\text { Dosage of } \\
\text { Soframycin (All } \\
\text { Subconjunctival) }\end{array}$ & $\begin{array}{l}\text { Result of } \\
\text { Treatment }\end{array}$ \\
\hline 1 & $\begin{array}{l}\text { Corneal Ulcer } \\
\text { (spontaneous) } \\
1 \mathrm{~mm} . \text { hypopyon }\end{array}$ & B. coli. & $\begin{array}{l}250 \mathrm{mg} \text {. } \\
\text { Single dose }\end{array}$ & $\begin{array}{l}\text { Rapid initial } \\
\text { improvement } \\
\text { Healed in } 3 \text { days }\end{array}$ \\
\hline 2 & $\begin{array}{l}\text { Corneal Ulcer } \\
\text { (spontaneous) } \\
2 \mathrm{~mm} \text {. hypopyon }\end{array}$ & No growth & $\begin{array}{l}250 \mathrm{mg} . \\
\text { Single dose }\end{array}$ & $\begin{array}{l}\text { Rapid improvement } \\
\text { Healed in } 4 \text { days }\end{array}$ \\
\hline 3 & $\begin{array}{l}\text { Infected Corneal Wound } \\
\text { (following foreign body) } \\
2 \mathrm{~mm} \text {. hypopyon }\end{array}$ & $\begin{array}{l}\text { Staph. aureus } \\
\text { Coagulase } \\
\text { positive }\end{array}$ & $\begin{array}{l}250 \mathrm{mg} . \text { on three } \\
\text { successive days } \\
(750 \mathrm{mg} . \text { total })\end{array}$ & Healed in 4 days \\
\hline 4 & $\begin{array}{l}\text { Corneal Ulcer } \\
\text { (spontaneous) } \\
2 \text { mm. hypopyon }\end{array}$ & No growth & $\begin{array}{l}100 \mathrm{mg} \text {. on two } \\
\text { successive days } \\
(200 \mathrm{mg} . \text { total })\end{array}$ & $\begin{array}{l}\text { Gradual improvement } \\
\text { Healed in } 5 \text { days }\end{array}$ \\
\hline 5 & $\begin{array}{l}\text { Corneal Ulcer } \\
\text { (spontaneous) } \\
\text { In senile patient } \\
2 \mathrm{~mm} \text {. hypopyon }\end{array}$ & $\begin{array}{l}\text { Staph. albus } \\
\text { Coagulase } \\
\text { negative }\end{array}$ & $\begin{array}{l}250 \mathrm{mg} \text {. on two } \\
\text { successive days } \\
(500 \mathrm{mg} . \text { total })\end{array}$ & $\begin{array}{l}\text { No immediate } \\
\text { improvement } \\
\text { Gradually settled }\end{array}$ \\
\hline 6 & $\begin{array}{l}\text { Corneal Ulcer } \\
\text { (spontaneous) } \\
1 \text { mm. hypopyon }\end{array}$ & Pneumococcus & $\begin{array}{l}250 \mathrm{mg} . \text { on two } \\
\text { successive days } \\
(500 \mathrm{mg} . \text { total })\end{array}$ & Healed in 3 days \\
\hline 7 & $\begin{array}{l}\text { Infected Corneal Wound } \\
\text { (following foreign body) }\end{array}$ & $\begin{array}{l}\text { Staph. aureus } \\
\text { Coagulase } \\
\text { positive }\end{array}$ & $\begin{array}{l}250 \mathrm{mg} . \text { on three } \\
\text { successive days } \\
(750 \mathrm{mg} . \text { total })\end{array}$ & $\begin{array}{l}\text { Marked improvement } \\
\text { after second dose } \\
\text { Healed in } 4 \text { days }\end{array}$ \\
\hline 8 & $\begin{array}{l}\text { Corneal Ulcer } \\
\text { (spontaneous) } \\
\text { Large area }\end{array}$ & No growth & $\begin{array}{l}200 \mathrm{mg} \text {. on two } \\
\text { successive days } \\
(400 \mathrm{mg} . \text { total })\end{array}$ & $\begin{array}{l}\text { Rapid initial } \\
\text { improvement, then } \\
\text { slow resolution over } \\
2 \text { weeks }\end{array}$ \\
\hline 9 & $\begin{array}{l}\text { Corneal Ulcer } \\
\text { (spontaneous) } \\
3 \mathrm{~mm} . \text { hypopyon }\end{array}$ & No growth & $\begin{array}{l}500 \mathrm{mg} \text {. } \\
\text { Single dose }\end{array}$ & $\begin{array}{l}\text { Marked improvement } \\
\text { after } 24 \text { hrs } \\
\text { Healed in } 3 \text { days }\end{array}$ \\
\hline 10 & $\begin{array}{l}\text { Corneal Wound } \\
\text { (penetrating) } \\
\text { Corneal abscess and } \\
\text { hypopyon }\end{array}$ & No growth & $\begin{array}{l}500 \mathrm{mg} . \\
\text { Single dose }\end{array}$ & $\begin{array}{l}\text { Infection unchecked } \\
\text { Ring abscess of } \\
\text { cornea developed }\end{array}$ \\
\hline 11 & $\begin{array}{l}\text { Infected Corneal Wound } \\
\text { (following foreign body) }\end{array}$ & No growth & $\begin{array}{l}500 \mathrm{mg} \text {. } \\
\text { Single dose }\end{array}$ & Healed in $24 \mathrm{hrs}$ \\
\hline 12 & $\begin{array}{l}\text { Infected Corneal Wound } \\
\text { (following foreign body) }\end{array}$ & B. xerosis & $\begin{array}{l}\left.\begin{array}{l}500 \mathrm{mg} . \\
\text { (first dose) } \\
300 \mathrm{mg} . \\
\begin{array}{l}\text { (second } \\
\text { dose) }\end{array}\end{array}\right\} 800 \\
\mathrm{mg} .\end{array}$ & Healed in 3 days \\
\hline 13 & $\begin{array}{l}\text { Infected Corneal Wound } \\
\text { (following foreign body) } \\
2 \mathrm{~mm} \text {. hypopyon }\end{array}$ & Pneumococcus & $\begin{array}{l}500 \mathrm{mg} . \\
\text { Single dose }\end{array}$ & $\begin{array}{l}\text { Marked improvement } \\
\text { after } 12 \text { hrs } \\
\text { Healed in } 3 \text { days }\end{array}$ \\
\hline
\end{tabular}


contin.

\begin{tabular}{|c|c|c|c|c|}
\hline $\begin{array}{l}\text { Case } \\
\text { No. }\end{array}$ & Nature of Case & Bacteriology & $\begin{array}{c}\text { Dosage of } \\
\text { Soframycin (All } \\
\text { Subconjunctival) }\end{array}$ & $\begin{array}{l}\text { Result of } \\
\text { Treatment }\end{array}$ \\
\hline 14 & $\begin{array}{l}\text { Infected Corneal Wound } \\
\text { (following foreign body) }\end{array}$ & Ps. pyocyanea & $\begin{array}{l}500 \mathrm{mg} \text {. on two } \\
\text { successive days } \\
\text { (1 g. total) }\end{array}$ & $\begin{array}{l}\text { Infection checked } \\
\text { Slow healing } \\
\text { subsequently }\end{array}$ \\
\hline 15 & $\begin{array}{l}\text { Infected Corneal Wound } \\
\text { (following foreign body) }\end{array}$ & No growth & $\begin{array}{l}500 \mathrm{mg} \text {. } \\
\text { Single dose }\end{array}$ & Healed in $24 \mathrm{hrs}$ \\
\hline 16 & $\begin{array}{l}\text { Corneal Abscess } \\
\text { (unknown aetiology) } \\
\text { In senile patient }\end{array}$ & B. xerosis & $\begin{array}{l}500 \mathrm{mg} \text {. } \\
\text { Single dose }\end{array}$ & $\begin{array}{l}\text { Rapid initial } \\
\text { improvement } \\
\text { Slow healing }\end{array}$ \\
\hline 17 & $\begin{array}{l}\text { Infected Corneal Wound } \\
\text { (following foreign body) }\end{array}$ & No growth & $\begin{array}{l}500 \mathrm{mg} \text {. } \\
\text { Single dose }\end{array}$ & Healed in $48 \mathrm{hrs}$ \\
\hline 18 & $\begin{array}{l}\text { Infected Corneal Wound } \\
\text { (following foreign body) }\end{array}$ & No growth & $\begin{array}{l}500 \mathrm{mg} \text {. } \\
\text { Single dose }\end{array}$ & Healed in $24 \mathrm{hrs}$ \\
\hline 19 & $\begin{array}{l}\text { Infected Corneal Wound } \\
\text { (following foreign body) }\end{array}$ & B. xerosis & $\begin{array}{l}500 \mathrm{mg} \text {. } \\
\text { Single dose }\end{array}$ & Healed in $24 \mathrm{hrs}$ \\
\hline 20 & $\begin{array}{l}\text { Infected Corneal Wound } \\
\text { (following foreign body) }\end{array}$ & No growth & $\begin{array}{l}500 \mathrm{mg} \text {. } \\
\text { Single dose }\end{array}$ & Healed in $24 \mathrm{hrs}$ \\
\hline 21 & $\begin{array}{l}\text { Infected Corneal Wound } \\
\text { (following foreign body) }\end{array}$ & No growth & $\begin{array}{l}500 \mathrm{mg} \text {. } \\
\text { Single dose }\end{array}$ & Healed in $24 \mathrm{hrs}$ \\
\hline 22 & $\begin{array}{l}\text { Corneal Ulcer } \\
\text { (unknown aetiology) } \\
\text { Recurrent }\end{array}$ & No growth & $\begin{array}{l}500 \mathrm{mg} \text {. } \\
\text { Single dose }\end{array}$ & $\begin{array}{l}\text { Rapid improvement } \\
\text { proceeding to } \\
\text { healing in } 3 \text { days }\end{array}$ \\
\hline 23 & $\begin{array}{l}\text { Infected Corneal Wound } \\
\text { (following foreign body) }\end{array}$ & No growth & $\begin{array}{l}500 \mathrm{mg} \text {. } \\
\text { Single dose }\end{array}$ & Healed in 2 days \\
\hline 24 & $\begin{array}{l}\text { Corneal Abscess } \\
\text { (following foreign body) }\end{array}$ & No growth & $\begin{array}{l}500 \mathrm{mg} \text {. } \\
\text { Single dose }\end{array}$ & $\begin{array}{l}\text { Rapid initial } \\
\text { improvement } \\
\text { Gradually healing } \\
\text { over } 10 \text { days }\end{array}$ \\
\hline 25 & $\begin{array}{l}\text { Infected Corneal Wound } \\
\text { (probably abrasion) } \\
1 \mathrm{~mm} \text {. hypopyon }\end{array}$ & No growth & $\begin{array}{l}300 \mathrm{mg} \text {. } \\
\text { Single dose }\end{array}$ & Rapid improvement \\
\hline 26 & $\begin{array}{l}\text { Corneal Ulcer } \\
\text { Recurrent }\end{array}$ & No growth & $\begin{array}{l}500 \mathrm{mg} . \\
\text { Single dose }\end{array}$ & $\begin{array}{l}\text { Infection checked } \\
\text { Gradually healing } \\
\text { subsequently }\end{array}$ \\
\hline 27 & $\begin{array}{l}\text { Infected Corneal Wound } \\
\text { (following foreign body) }\end{array}$ & No growth & $\begin{array}{l}500 \mathrm{mg} . \\
\text { Single dose }\end{array}$ & Healed after $24 \mathrm{hrs}$ \\
\hline 28 & $\begin{array}{l}\text { Corneal Abscess } \\
\text { (unknown aetiology) }\end{array}$ & No growth & $\begin{array}{l}500 \mathrm{mg} \text {. } \\
\text { Single dose }\end{array}$ & Rapid resolution \\
\hline 29 & $\begin{array}{l}\text { Corneal Ulcer } \\
\text { (case of spring catarrh) } \\
\text { Hypopyon }\end{array}$ & No growth & $\begin{array}{l}500 \mathrm{mg} \text {. } \\
\text { Single dose }\end{array}$ & $\begin{array}{l}\text { Rapid improvement } \\
\text { after injection } \\
\text { Slow resolution }\end{array}$ \\
\hline 30 & $\begin{array}{l}\text { Infected Corneal Wound } \\
\text { (following foreign body) }\end{array}$ & $\begin{array}{l}\text { Staph. aureus } \\
\text { Coagulase } \\
\text { positive }\end{array}$ & $\begin{array}{l}500 \mathrm{mg} . \\
\text { Single dose }\end{array}$ & $\begin{array}{l}\text { Infection progressed } \\
\text { unchecked }\end{array}$ \\
\hline
\end{tabular}




\section{Results}

The results of the treatment of corneal infections by the subconjunctival administration of Soframycin were mostly satisfactory. Of the thirty cases treated, 21 improved rapidly and seven others more slowly. Two patients did not appear to benefit.

In the majority of cases the clinical appearance of dense, localized corneal infiltration suggested that staphylococci or pneumococci were the causative organisms. Two cases of severe hypopyon corneal ulceration from which pneumococci were isolated healed rapidly after Soframycin treatment. Another case of severe hypopyon ulcer (Case 12), which had failed to improve despite the subconjunctival administration of 500,000 units penicillin and $0.5 \mathrm{~g}$. streptomycin healed rapidly after Soframycin therapy. It should be noted, however, that in one instance (Case 30), where Soframycin had failed to arrest the infection, the subconjunctival injection of Crystamycin (penicillin and streptomycin) was effective. In four cases the clinical appearance was that of diffuse spreading necrosis characteristic of infections due to the Gram-negative bacilli. Three of these, including one (Case 14) where there was cultural confirmation of the presence of Ps. pyocyanea, responded satisfactorily to Soframycin. In the remaining case in this group (Case 10), where infection followed a perforating corneal wound, Soframycin was ineffective, but the infection was controlled by the subconjunctival administration of streptomycin and Polymyxin.

In no case was there any untoward reaction following the subconjunctival injection of Soframycin and little pain was caused by the injections. It was not found necessary to add a local anaesthetic to the solvent; the topical application of 4 per cent. cocaine drops to the conjunctival sac provided satisfactory anaesthesia.

\section{Summary}

(1) Thirty cases of corneal infection treated by the subconjunctival injection of Soframycin are described. Satisfactory results were obtained in the majority.

(2) Soframycin is easily soluble, and $500 \mathrm{mg}$. dissolved in $1 \mathrm{ml}$. water may be injected subconjunctivally with safety.

(3) Soframycin is almost completely non-irritant and little pain follows subconjunctival injection.

We should like to thank the surgeons of Moorfields Eye Hospital who allowed us to treat their patients. We are also greatly indebted to members of the Departments of Pathology of Moorfields Eye Hospital and the Middlesex Hospital who undertook the bacteriological examinations.

\section{REFERENCES}

Arnslie, D., and Henderson, W. G. (1958). Brit. J. Ophthal., 42, 513. Massenat-Derouche, B. (1954). M.D. Thesis (Paris). 